Cite this: Mol. BioSyst., 2014, 10, 103

Received 17th June 2013, Accepted 2nd October 2013

www.rsc.org/molecularbiosystems

\section{Size of quorum sensing communities $\dagger$}

\author{
Jesper Ferkinghoff-Borg*a and Thomas Sams ${ }^{\star b}$
}

\begin{abstract}
Ensembles of bacteria are able to coordinate their phenotypic behavior in accordance with the size, density, and growth state of the ensemble. This is achieved through production and exchange of diffusible signal molecules in a cell-cell regulatory system termed quorum sensing. In the generic quorum sensor a positive feedback in the production of signal molecules defines the conditions at which the collective behavior switches on. In spite of its conceptual simplicity, a proper measure of biofilm colony "size" appears to be lacking. We establish that the cell density multiplied by a geometric factor which incorporates the boundary conditions constitutes an appropriate size measure. The geometric factor is the square of the radius for a spherical colony or a hemisphere attached to a reflecting surface. If surrounded by a rapidly exchanged medium, the geometric factor is divided by three. For a diskshaped biofilm the geometric factor is the horizontal dimension multiplied by the height, and the square of the height of the biofilm if there is significant flow above the biofilm. A remarkably simple factorized expression for the size is obtained, which separates the all-or-none ignition caused by the positive feedback from the smoother activation outside the switching region.
\end{abstract}

\section{Introduction}

Quorum sensing (QS) is a cell-cell signaling system used by bacteria to keep track of the size, density, and the growth state of the population. The regulation depends on the concentration of diffusible signal molecules which are typically small peptides for Gram positives and acyl homoserine lactones for Gram negatives. The most basic regulatory system consists of a signal molecule synthetase and a transcriptional regulator as illustrated in Fig. 1. At low population "size" the signal molecules are produced at the background level. At higher population "size", the signal molecules accumulate and activate the transcriptional regulator to induce further transcription of the signal molecule synthetase. This positive feedback loop results in a rapid amplification of the signal. ${ }^{1-4}$ In these systems the regulator $\mathrm{R}$ is produced constitutively and is activated through binding of signal molecules $\mathrm{S}$. The activated regulator concentration

$$
r_{\mathrm{a}}=\left[\mathrm{R}_{2} \mathrm{~S}_{2}\right]
$$

is the intrinsic measure of whether a quorum is formed. This intracellular concentration controls the quorum sensing feedback as well as the transcription of specific quorum regulated

\footnotetext{
${ }^{a}$ Center for Biological Sequence Analysis, Dept. of Systems Biology, Technical University of Denmark, DK-2800, Denmark.E-mail: jesperfb@cbs.dtu.dk

${ }^{b}$ Biomedical Engineering, Department of Electrical Engineering, Technical University of Denmark, DK-2800 Lyngby, Denmark.E-mail: tsams@dtu.dk $\dagger$ Electronic supplementary information (ESI) available. See DOI: 10.1039/ c3mb70230h
}
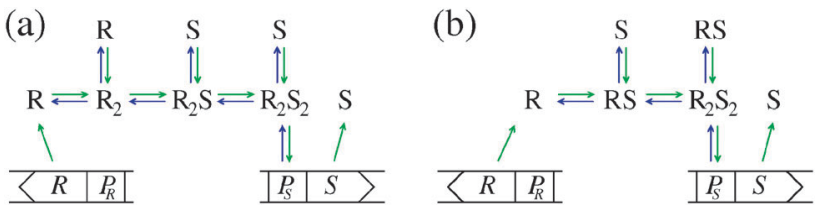

Fig. 1 Schematic examples of pathways in regulator $(R)$ induction and signal (S) binding that may lead to static activated dimer $\left(\mathrm{R}_{2} \mathrm{~S}_{2}\right)$ concentrations of the form (2) or (4). $R, P_{R}$ and $S, P_{S}$ denote the gene and promoter region for the regulator and the signal molecule synthetase respectively. (a) Dimerization drives ligand binding. (b) Ligand binding drives dimerization.

target genes. Following this generic design of quorum sensors in Gram negative bacteria we derive a simple expression for the size of a biofilm colony and demonstrate how a single quorum sensor depends on density, geometry, boundary conditions, and maximal regulator concentration. The maximal regulator concentration, in turn, may be sensitive to specific conditions for the growth state required for the quorum conditions to be satisfied.

The collective behavior displayed in QS was first reported in Vibrio fischeri where it activates the production of light in large cell colonies. ${ }^{1-3}$ Quorum sensing systems have been reported in many bacterial systems including Aeromonas hydrophila, ${ }^{5-7}$ Agrobacterium tumefaciens, ${ }^{8}$ and Pseudomonas aeruginosa. ${ }^{9,10}$

It is our hope that the simple factorized expression for the size of a quorate community presented here will provide a more general guidance on how to speak of the size of a bacterial quorum. In particular we note that boundary conditions and geometrical shape are integral parts of the "size" of the colony. 
Table 1 Variables and parameters used in the model

\begin{tabular}{|c|c|c|}
\hline Variable & Details & Explanation \\
\hline$P_{R}$ & & Promoter for R synthetase \\
\hline $\mathrm{R}$ & & Regulator protein \\
\hline$P_{S}$ & & Promoter for S synthetase \\
\hline $\mathrm{S}$ & & Signal molecule/ligand \\
\hline$s$ & {$[\mathrm{~S}]$} & Signal molecule concentration \\
\hline$b_{\mathrm{s}}$ & & Background S production \\
\hline$k_{\mathrm{s}}$ & $\sim 100 b_{\mathrm{s}}$ & Maximal induced S production \\
\hline$\kappa_{\mathrm{s}}$ & $b_{\mathrm{s}}<\kappa_{\mathrm{s}}<k_{\mathrm{s}}$ & Induced S production \\
\hline$K$ & $10 \mathrm{nM}-10 \mu \mathrm{M}$ & $\mathrm{R}_{2} \mathrm{~S}_{2}$ dissociation constant $\mathrm{t}^{12,14-16}$ \\
\hline$K_{\mathrm{s}}$ & & $\mathrm{R}_{2} \mathrm{~S}_{2}-P_{S}$ dissociation constant \\
\hline$r_{\mathrm{a}}$ & {$\left[\mathrm{R}_{2} \mathrm{~S}_{2}\right]$} & Activated regulator concentration \\
\hline$r_{\mathrm{m}}$ & $\gg \frac{b_{\mathrm{s}}}{k_{\mathrm{s}}} K_{\mathrm{s}}$ & Maximal $r_{\mathrm{a}}$ \\
\hline$h$ & $1-2$ & Regulator oligomerization order \\
\hline$D$ & $\sim 2 \mathrm{~mm}^{2} \mathrm{~h}^{-1}$ & Diffusion constant for S (AHLs) \\
\hline$\rho_{\mathrm{v}}$ & $<1$ & Bacterial density (v/v) \\
\hline$r$ & & Radial coordinate \\
\hline$z$ & & Height coordinate \\
\hline$\Sigma$ & & Colony "size" measure \\
\hline $\mathcal{R}_{\text {sphere }}$ & $10-70 \mu \mathrm{m}$ & Radius of colony ${ }^{17,18}$ \\
\hline $\mathcal{R}_{\text {disk }}$ & $10-500 \mu \mathrm{m}$ & Radius of colony ${ }^{17}$ \\
\hline $\mathcal{H}$ & $10-70 \mu \mathrm{m}$ & Height of colony ${ }^{17,19,20}$ \\
\hline $\mathcal{L}$ & $2-7 \mathrm{~mm}$ & Degradation length (AHLs pH 8, 7) \\
\hline$\lambda_{\mathrm{s}}$ & $0.03-0.25 \mathrm{~h}^{-1}$ & S degradation (AHLs, pH 7, 8) \\
\hline
\end{tabular}

All variables used in the manuscript are summarized in Table 1 while the size measures for different geometries and boundary conditions are summarized in Table 2 .

\section{Model}

\subsection{Self consistent description}

Typical motifs for activation of regulators involve dimerization as indicated in Fig. 1a where dimerization drives ligand binding and in Fig. 1b where ligand binding drives dimerization. In the quasistatic limit, the concentration of activated regulator may be parametrized as a product of a signal molecule switch of order $h$ and a maximal level $r_{\mathrm{m}}$

$$
r_{\mathrm{a}}=\frac{s^{h}}{K^{h}+s^{h}} r_{\mathrm{m}}
$$

where $K$ is the effective dissociation constant for binding of the signal molecules to the regulator. ${ }^{11}$ With $h=2$ this describes

Table 2 Size measures at the center of the colony for a sphere and for a disk resting on a signal molecule reflecting surface. The radius of the colony is $\mathcal{R}$ and the height of the disk is $\mathcal{H}$. When the degradation rate $\lambda_{\mathrm{s}}$ of signal molecules is significant, i.e. when the length $\mathcal{L}=\sqrt{D / \lambda_{\mathrm{s}}}$ is smaller than one or more of the geometrical dimensions of the colony, this dimension is (approximately) replaced by $\mathcal{L}$ in the size measure. Eventually all geometrical sizes disappear and the size, $\Sigma$, then measures the cell density

\begin{tabular}{llll}
\hline & $\mathcal{H} \ll \mathcal{R} \ll \mathcal{L}$ & $\mathcal{H} \ll \mathcal{L} \ll \mathcal{R}$ & $\mathcal{L} \ll \mathcal{H} \ll \mathcal{R}$ \\
Geometry, boundary & $\Sigma$ & $\Sigma$ & $\Sigma$ \\
\hline Sphere, open & $\mathcal{R}^{2} \rho_{\mathrm{v}}$ & $2 \mathcal{L}^{2} \rho_{\mathrm{v}}$ & $2 \mathcal{L}^{2} \rho_{\mathrm{v}}$ \\
Sphere, absorbing & $\frac{1}{3} \mathcal{R}^{2} \rho_{\mathrm{v}}$ & $2 \mathcal{L}^{2} \rho_{\mathrm{v}}$ & $2 \mathcal{L}^{2} \rho_{\mathrm{v}}$ \\
Disk, open & $2 \mathcal{R} \mathcal{H} \rho_{\mathrm{v}}$ & $2 \mathcal{L} \mathcal{H} \rho_{\mathrm{v}}$ & $2 \mathcal{L}^{2} \rho_{\mathrm{v}}$ \\
Disk, absorbing & $\mathcal{H}^{2} \rho_{\mathrm{v}}$ & $\mathcal{H}^{2} \rho_{\mathrm{v}}$ & $2 \mathcal{L}^{2} \rho_{\mathrm{v}}$
\end{tabular}

dimerization followed by cooperative binding of signal molecules. Solving for the ligand concentration we get

$$
s=\frac{r_{\mathrm{a}}^{1 / h}}{\left(r_{\mathrm{m}}-r_{\mathrm{a}}\right)^{1 / h}} K
$$

Another form of interest would be

$$
\begin{aligned}
& r_{\mathrm{a}}=\frac{s^{h}}{(K+s)^{h}} r_{\mathrm{m}} \\
& s=\frac{r_{\mathrm{a}}^{1 / h}}{r_{\mathrm{m}}^{1 / h}-r_{\mathrm{a}}^{1 / h}} K
\end{aligned}
$$

which covers non-cooperative binding of signal molecules as well as typical cases where signal molecule binding drives dimerization. ${ }^{11,12}$ With $h=1$ we have monomer behavior. Though this is not a typical motif in quorum sensors, it is included to illustrate the advantage of designs involving oligomers to achieve the hysteresis leading to robust all-or-none switching seen in many positive feedback systems. ${ }^{13}$

The binding of the activated regulator to the signal promoter site leads to increased expression of the signal molecule synthetase. In the quasistatic limit the resulting signal molecule production can be expressed

$$
\kappa_{\mathrm{s}}=\frac{K_{\mathrm{s}}}{K_{\mathrm{s}}+r_{\mathrm{a}}} b_{\mathrm{s}}+\frac{r_{\mathrm{a}}}{K_{\mathrm{s}}+r_{\mathrm{a}}} k_{\mathrm{s}}=\frac{\frac{b_{\mathrm{s}}}{k_{\mathrm{s}}} K_{\mathrm{s}}+r_{\mathrm{a}}}{K_{\mathrm{s}}+r_{\mathrm{a}}} k_{\mathrm{s}}
$$

normalized as produced number per time per intracellular volume. This is merely a shift from a low background production $b_{\mathrm{s}}$ when $r_{\mathrm{a}}$ is below $\frac{b_{\mathrm{s}}}{k_{\mathrm{s}}} K_{\mathrm{s}}$ to the higher, active, production $k_{\mathrm{s}}$ when the concentration $r_{\mathrm{a}}$ is above $K_{\mathrm{s}}$. Note that the feedback mechanism turns on at the concentration

$$
r_{\mathrm{a}} \sim \frac{b_{\mathrm{s}}}{k_{\mathrm{s}}} K_{\mathrm{s}}
$$

which needs to be a couple of orders of magnitude lower than the dissociation constant $K_{\mathrm{s}}$ for the feedback system to produce a pronounced switch.

The production of signal molecules and their intercellular diffusion are linked together in the diffusion equation. Given a production rate $\kappa_{\mathrm{s}}(r)$ per intracellular volume and a volume fraction $\rho_{\mathrm{v}}(r)$ occupied by cells, the diffusion equation with the source term reads

$$
\frac{\partial s}{\partial t}=D \Delta s+\rho_{\mathrm{v}} \kappa_{\mathrm{s}}
$$

where $D$ is the diffusion constant for the signal molecules and $\Delta=\nabla^{2}=\frac{\partial^{2}}{\partial x^{2}}+\frac{\partial^{2}}{\partial y^{2}}+\frac{\partial^{2}}{\partial z^{2}}$ is the Laplace operator. Since single cells are unable to ignite or even maintain an ignited feedback loop the net contribution from binding and dissociation of signal molecules to regulators could be ignored when writing eqn (8). (Active transport of signal molecules across the cell membrane which, to lowest order, leads to a rescaling between 
the intracellular and extracellular ligand concentration was not included.) At the steady state

$$
\frac{1}{2} \Delta s=-\frac{\rho_{\mathrm{v}}}{2 D} \kappa_{\mathrm{s}}
$$

which we shall use to establish qualitative expressions for the conditions required to form a quorum.

\subsection{Simple estimates}

As a starting point, let us solve eqn (9) for a spherical colony of bacteria with radius $\mathcal{R}$. This also solves the case of a hemispherical biofilm on a reflecting surface. The volume fraction occupied by cells, $\rho_{\mathrm{v}}$, is zero outside the biofilm and assumed constant inside the colony.

If the colony is very small or very large, we can assume that $\kappa_{\mathrm{s}}$ is constant and takes the values $b_{\mathrm{s}}$ or $k_{\mathrm{s}}$ respectively. Further, due to the feedback, the switching on/off is very abrupt which results in the whole colony being in the same state, i.e. $\kappa_{\mathrm{s}}$ can be assumed constant throughout the colony. This approximation is confirmed by numerical simulations below.

The solution of the steady state eqn (9) depends on the boundary condition. If the colony is surrounded by tissue or by non-flowing liquid, the boundary condition simply requires $s(r)$ and its derivative to be continuous across the boundary. This leads to

$$
s(r)=\frac{\kappa_{\mathrm{s}} \mathcal{R}^{2} \rho_{\mathrm{v}}}{2 D}\left\{\begin{array}{cc}
1-\frac{1}{3}\left(\frac{r}{\mathcal{R}}\right)^{2} & , r<\mathcal{R} \\
\frac{2}{3} \frac{\mathcal{R}}{r} & , r>\mathcal{R}
\end{array}\right.
$$

and the density of signal molecules at the center of the colony is

$$
s(0)=\frac{\kappa_{\mathrm{s}}}{2 D} \mathcal{R}^{2} \rho_{\mathrm{v}}
$$

Self consistency dictates that the autoinducer production rate $\kappa_{\mathrm{s}}$ in (11) be equal to the rate in (6). When the signal molecule concentration $s$ is approximately expressed in terms of $r_{\mathrm{a}}$ by (3), this leads to a fairly simple factorized expression for $\mathcal{R}^{2} \rho_{\mathrm{v}}$ of the colony

$$
\Sigma=\mathcal{R}^{2} \rho_{\mathrm{v}}=\frac{2 D K}{b_{\mathrm{s}}} \underbrace{\frac{b_{\mathrm{s}}}{k_{\mathrm{s}} \frac{K_{\mathrm{s}}+r_{\mathrm{a}}}{k_{\mathrm{s}}} K_{\mathrm{s}}+r_{\mathrm{a}}}}_{\text {feedback switch in }] \frac{b_{\mathrm{s}}}{k_{\mathrm{s}}} ; 1[} \underbrace{\left(\frac{r_{\mathrm{a}}}{r_{\mathrm{m}}-r_{\mathrm{a}}}\right)^{1 / h}}_{\text {forward activation in }] 0 ; \infty[}
$$

as a function of the activated regulator level $r_{\mathrm{a}}$. With $h=2$ eqn (12) describes quorum switching with cooperative binding of ligands to the regulator. The relevant "size" measure is seen to be $\Sigma=\mathcal{R}^{2} \rho_{\mathrm{v}}$, i.e. a combination of density and geometrical size of the biofilm. Notably, this size measure is a function of the activated dimer concentration only and can be expressed as a product of a normal forward activation and a feedback switch. Below we shall see that the r.h.s. is unaltered when the geometry and boundary conditions change. This means that all information regarding geometry, density, and boundary conditions is on the l.h.s. of the equation. In this sense $\Sigma=\mathcal{R}^{2} \rho_{\mathrm{v}}$ is a proper "size" measure. The forward activation and the feedback

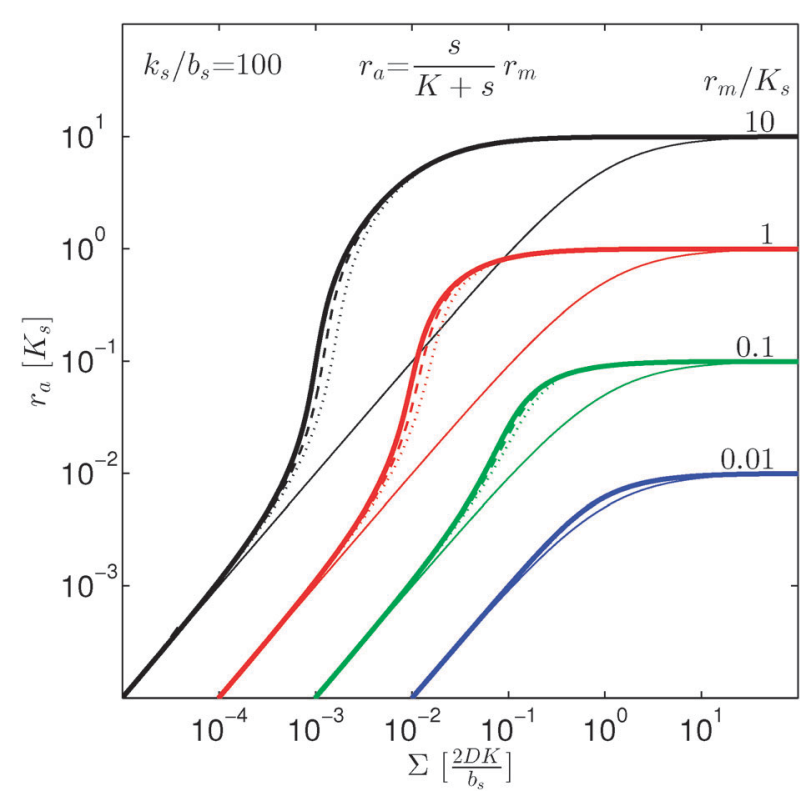

Fig. 2 Self consistent estimate of the concentration of the activated regulator at the center of a bacterial colony as a function of the colony size. The ligand binding is assumed to be first order. Thin lines indicate the $r_{\mathrm{a}}$ level for a first order ligand binding without feedback. The approximate solution is the same for all geometries. The size measure $\Sigma$ depends on the specific geometry and boundary conditions as indicated in Table 2 . At low regulator levels the feedback has no effect. At high regulator levels, the role of the feedback loop is to make the ignition of the QS system happen more abruptly. Dashed lines represent the numerical solution for a spherical colony with open boundary condition $\left(\Sigma=\mathcal{R}^{2} \rho_{\mathrm{v}}\right)$. Dotted curves are the numerical solution for a spherical colony with absorbing boundary $\left(\Sigma=\frac{1}{3} \mathcal{R}^{2} \rho_{\mathrm{v}}\right)$.

switch being dimensionless, the natural unit of size is seen to be $2 D K / b_{\mathrm{s}}$.

In Fig. 2 we see that for $h=1$ the size dependence does not lead to hysteresis and therefore is not a fully developed all-ornone switch. However, for $h=2$ seen in Fig. 3 the forward activation is sufficiently steep to ensure that the feedback switch leads to a robust all-or-none switch implied by the hysteresis. ${ }^{13}$

If we consider the case (4) where ligand bindings are independent we get instead

$$
\Sigma=\mathcal{R}^{2} \rho_{\mathrm{v}}=\frac{2 D K}{b_{\mathrm{s}}} \underbrace{\frac{b_{\mathrm{s}}}{k_{\mathrm{s}} \frac{K_{\mathrm{s}}+r_{\mathrm{a}}}{b_{\mathrm{s}}} K_{\mathrm{s}}+r_{\mathrm{a}}}}_{\text {feedback switch in }] \frac{b_{\mathrm{s}}}{k_{\mathrm{s}}} ; 1[} \quad \underbrace{\frac{r_{\mathrm{a}}^{1 / h}}{r_{\mathrm{m}}^{1 / h}-r_{\mathrm{a}}^{1 / h}}}_{\text {forward activation in }] 0 ; \infty[}
$$

with $h=2$. The size measure $\Sigma=\mathcal{R}^{2} \rho_{\mathrm{v}}$ remains unchanged.

As seen in Fig. 4, expressions (12) and (13) have similar behavior at the ignition point, $r_{\mathrm{a}} \sim \frac{b_{\mathrm{s}}}{k_{\mathrm{s}}} K_{\mathrm{s}}$, and have identical asymptotic behavior. It is therefore not critical to the ignition function of the feedback loop that consecutive ligand bindings are cooperative. However, the cooperativity extends the bistable region somewhat and therefore may aid in stabilizing the switch. 


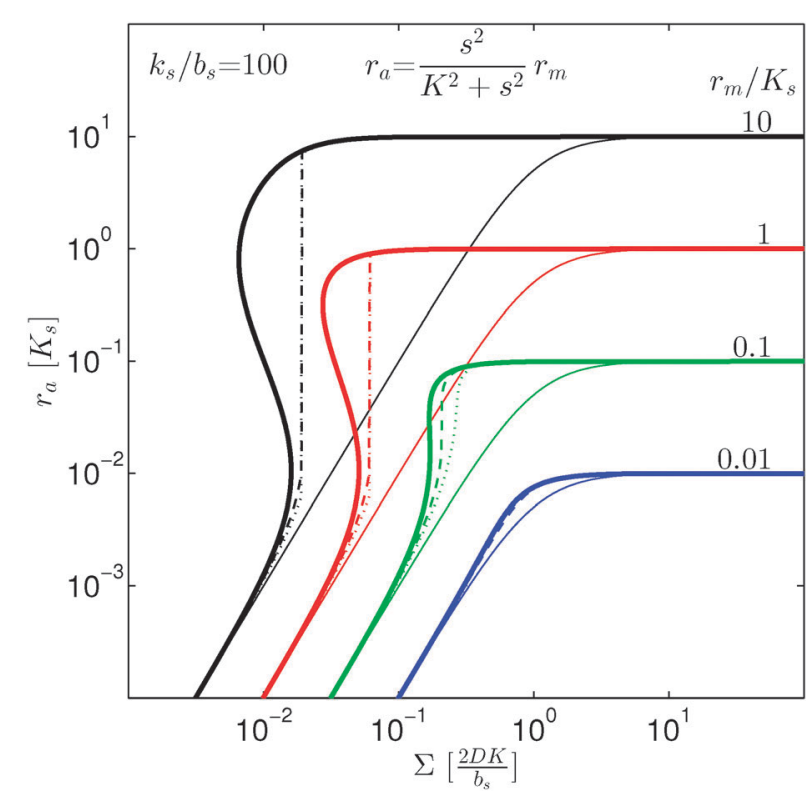

Fig. 3 Self consistent estimate of the concentration of the activated regulator at the center of a bacterial colony as a function of the colony size. Ligand binding is assumed to be second order and fully cooperative. Thin lines indicate the $r_{\mathrm{a}}$ level for a second order cooperative ligand binding without feedback. The size measure $\Sigma$ depends on the specific geometry and boundary conditions as indicated in Table 2. Dashed lines represent the numerical solution for open boundary condition $\left(\Sigma=\mathcal{R}^{2} \rho_{\mathrm{v}}\right)$. Dotted curves are the numerical solution with absorbing boundary $\left(\Sigma=\frac{1}{3} \mathcal{R}^{2} \rho_{\mathrm{v}}\right)$.

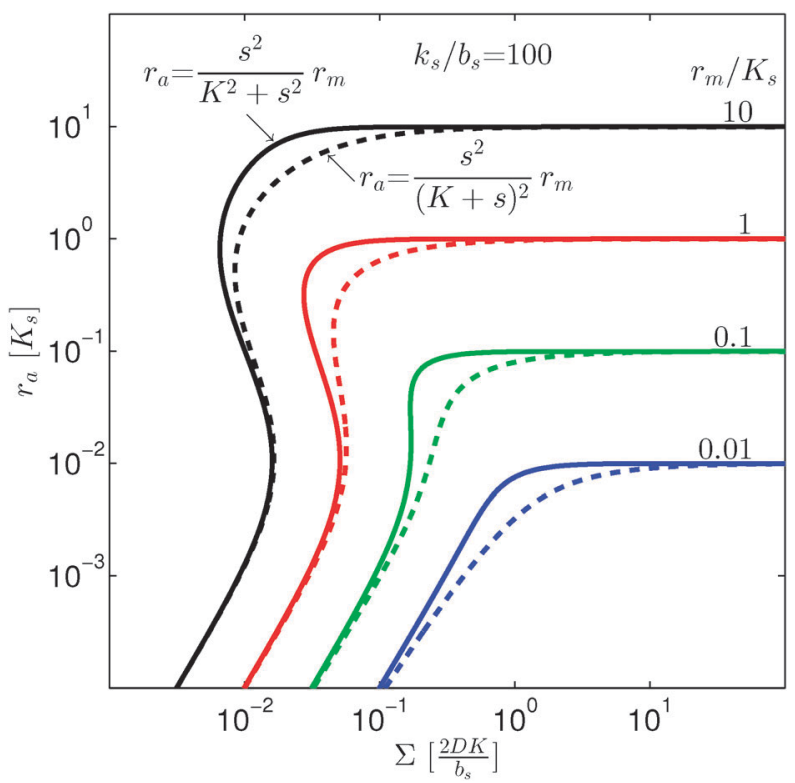

Fig. 4 Self consistent estimate of the concentration of the activated dimer regulator as a function of the bacterial colony size. The full curves represent the case of fully cooperative ligand binding and the dashed curves represent the independent binding of ligands. When $r_{\mathrm{m}} \gtrsim K_{\mathrm{s}}$ the hysteresis leading to a robust switch is present for cooperative ligand binding as well as for independent binding. The cooperative binding makes the hysteresis accessible at lower $r_{\mathrm{m}}$.

When growth conditions result in a maximal regulator concentration $r_{\mathrm{m}}$ smaller than $\frac{b_{\mathrm{s}}}{k_{\mathrm{s}}} K_{\mathrm{s}}$, it is impossible to ignite the feedback loop no matter how high the concentration of signal molecules is. When growth conditions lead to a maximal dimer level significantly higher than $\frac{b_{\mathrm{s}}}{k_{\mathrm{s}}} K_{\mathrm{s}}$ the feedback loop can be ignited. The effect of the feedback is an increased production of signal molecules and an abrupt increase in $r_{\mathrm{a}}$. Even for first order ligand binding $(h=1)$ the feedback makes the transition to higher $r_{\mathrm{a}}$ levels happen quite abruptly as a function of colony "size" as seen in Fig. 2. For the more typical second-order ligand binding $(h=2)$ shown in Fig. 3 the feedback displays a second order transition, i.e. the transition is practically a Heaviside step function with $r_{\mathrm{a}}$ jumping more than a factor $k_{\mathrm{s}} / b_{\mathrm{s}}$ at the quorum ignition point.

At size, $\Sigma$, larger than $2 D K / b_{\mathrm{s}}$ the feedback loop has no effect: the activated regulator concentration $r_{\mathrm{a}}$ is simply equal to the maximal regulator concentration $r_{\mathrm{m}}$. In such large biofilm communities $r_{\mathrm{a}}$ is therefore completely determined by the growth state.

With absorbing boundary condition

$$
s(r)=\frac{1}{3} \frac{\kappa_{\mathrm{s}} \mathcal{R}^{2} \rho_{\mathrm{v}}}{2 D}\left\{\begin{array}{cc}
1-\left(\frac{r}{\mathcal{R}}\right)^{2} & , r<\mathcal{R} \\
0 & , r>\mathcal{R}
\end{array}\right.
$$

which leads to the approximate size expression

$$
\Sigma=\frac{1}{3} \mathcal{R}^{2} \rho_{\mathrm{v}}=\frac{2 D K}{b_{\mathrm{s}}} \underbrace{\frac{b_{\mathrm{s}}}{k_{\mathrm{s}}} \frac{K_{\mathrm{s}}+r_{\mathrm{a}}}{\frac{b}{\mathrm{~s}}_{\mathrm{s}} K_{\mathrm{s}}+r_{\mathrm{a}}}}_{\text {feedback switch in }] \frac{b_{\mathrm{s}}}{k_{\mathrm{s}}} ; 1[} \underbrace{\left(\frac{r_{\mathrm{a}}}{r_{\mathrm{m}}-r_{\mathrm{a}}}\right)^{1 / h}}_{\text {forward activation in }] 0 ; \infty[}
$$

This means that it only takes a three times larger $\mathcal{R}^{2} \rho_{\mathrm{v}}$ to reach the quorum sensing ignition level with absorbing boundary condition than with free boundary.

For a thin biofilm with height $\mathcal{H}$ growing on a reflecting surface and covered by rapidly exchanged liquid we get

$$
s(z)=\frac{\kappa_{\mathrm{s}} \mathcal{H}^{2} \rho_{\mathrm{v}}}{2 D}\left(1-\left(\frac{z}{\mathcal{H}}\right)^{2}\right)
$$

where $z$ is the height above the surface. Using the value at the bottom we get the size measure

$$
\Sigma=\mathcal{H}^{2} \rho_{\mathrm{v}}
$$

for the ignition.

If the liquid above the biofilm is not moving we get instead

$$
s(0)=2 \frac{\kappa_{\mathrm{s}} \mathcal{R H} \rho_{\mathrm{v}}}{2 D}
$$

at the bottom of the biofilm and correspondingly the size measure

$$
\Sigma=2 \mathcal{R} \mathcal{H} \rho_{\mathrm{v}}
$$

where $\mathcal{R}$ is the radius of the biofilm. (Details of the calculation are given in the ESI $\dagger$ and ref. 33.)

In all cases the scaling properties (r.h.s. of (12)) are conserved. Thus, the interpretation of "size" depends critically on 
the geometry of the assembly of cells. We note that the relevant geometry for different types of growth is dictated by the environmental conditions. The disk geometry is relevant when a biofilm grows as a thin film on a surface ${ }^{17,19,20}$ whereas the spherical geometry is relevant for a more bulky type of growth. ${ }^{18,19}$

\subsection{Signal molecule degradation}

In significantly alkaline environments signal molecules degrade $^{21-23}$ which suggests the inclusion of a negative term proportional to the signal molecule concentration. Let us consider how this changes our conclusions. The diffusion equation now reads

$$
\frac{\partial s}{\partial t}=D \Delta s-\lambda_{\mathrm{s}} s+\rho_{\mathrm{v}} \kappa_{\mathrm{s}}
$$

where $\lambda_{\mathrm{s}}$ is the degradation rate. Dimensional analysis tells us that loss from degradation competes with loss from diffusion at system dimensions around the characteristic length $\mathcal{L}=\sqrt{D / \lambda_{\mathrm{s}}}$. I.e. when $\mathcal{L}$ is much smaller than a system dimension, the system dimension should be replaced by the $\mathcal{L}$ in our size measures $\Sigma$.

In the simple case of an extended biofilm of height $\mathcal{H}$ the solution to (20) is

$$
s(z)=\frac{\kappa_{\mathrm{s}} \rho_{\mathrm{v}} \mathcal{L}^{2}}{2 D} \begin{cases}2-\mathrm{e}^{-\mathcal{H} / \mathcal{L}}\left(\mathrm{e}^{z / \mathcal{L}}+\mathrm{e}^{-z / \mathcal{L}}\right) & , z<\mathcal{H} \\ \left(\mathrm{e}^{\mathcal{H} / \mathcal{L}}-\mathrm{e}^{-\mathcal{H} / \mathcal{L}}\right) \mathrm{e}^{-z / \mathcal{L}} & , z>\mathcal{H}\end{cases}
$$

where $\mathcal{L}=\sqrt{D / \lambda_{\mathrm{s}}}$ is the characteristic length at which the degradation competes with diffusion. At the bottom of the biofilm, $z=0$, this leads to

$$
s(0)=\frac{\kappa_{\mathrm{s}} \rho_{\mathrm{v}}}{2 D} \begin{cases}2 \mathcal{L}^{2} & , \mathcal{L} \ll \mathcal{H} \\ 2 \mathcal{L} \mathcal{H} & , \mathcal{L} \gg \mathcal{H}\end{cases}
$$

Comparison to (11) and (18) tells us that whenever $\mathcal{L}$ is smaller than a geometrical dimension, the geometrical dimension should be replaced by $\mathcal{L}$ (within a factor of $\sqrt{2}$ ). Table 2 summarizes the size measures for different geometries and also includes the modifications following from degradation.

\subsection{Numerical solution}

We saw that even with constant $\kappa_{\mathrm{s}}$ throughout the colony the signal molecule concentration $s$ drops to $\frac{2}{3}$ of the central value at the edge of the colony. If the colony is surrounded by rapidly flowing liquid, the boundary value drops to zero. Since $\kappa_{\mathrm{s}}$ and $s$ are intertwined it is therefore reasonable to check the validity of keeping $\kappa_{\mathrm{s}}$ constant (all or none switch). This is now done in a numerical solution. After inserting (2) and (6) in (9) we arrive at the self consistent equation

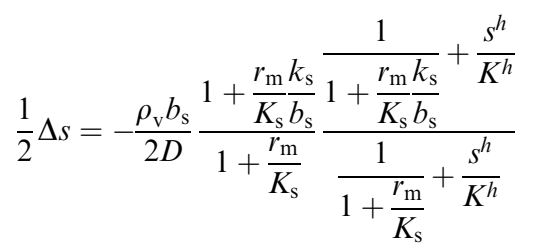

Changing units $r_{\mathrm{m}} / K_{\mathrm{s}} \rightarrow r_{\mathrm{m}}, k_{\mathrm{s}} / b_{\mathrm{s}} \rightarrow k_{\mathrm{s}}, s / K \rightarrow s$, and $r / \mathcal{R} \rightarrow r$ eqn (23) becomes

$$
\frac{1}{2 r^{2}} \frac{\partial}{\partial r}\left(r^{2} \frac{\partial s}{\partial r}\right)=-\frac{\mathcal{R}^{2} \rho_{\mathrm{v}}}{2 D K / b_{\mathrm{s}}} \frac{1+r_{\mathrm{m}} k_{\mathrm{s}}}{1+r_{\mathrm{m}}} \frac{\frac{1}{1+r_{\mathrm{m}} k_{\mathrm{s}}}+s^{h}}{\frac{1}{1+r_{\mathrm{m}}}+s^{h}}
$$

with the boundary condition $\partial s(0) / \partial r=0$ at the origin. With open boundary the solution is proportional to $r^{-1}$ outside the colony. Since $s$ as well as the derivative are continuous across the boundary this gives the condition $\partial s(1) / \partial r=-s(1)$ at the boundary. For a biofilm surrounded by rapidly exchanged liquid we get instead the boundary condition $s(1)=0$.

The numerical solution, $s(r)$, is subsequently used to calculate $r_{\mathrm{a}}$ via (2). The values at the center are compared with the approximate expression in Fig. 2 for first order ligand binding. The approximate solution gives an excellent description of the transition from quiet to ignited quorum sensing. In Fig. 3 the numerical solutions at the center are compared to the approximate expression (12) for second order cooperative ligand binding. The second order ligand binding leads to a very abrupt ignition. In the bistable region the numerical solver was initialized with the static solution with no feedback in order to capture the ignition of the quorum sensor. Again, the "size" required to ignite the feedback is captured well by the simple model (12) and favors the approximation of keeping $\kappa_{\mathrm{s}}$ constant throughout the colony.

With absorbing boundary condition for the ligand concentration $\kappa_{\mathrm{s}}(r)$ approaching the $b_{\mathrm{s}}$ eventually. Thus, very near the edge, there is a small region where the approximation of keeping $\kappa_{\mathrm{s}}$ constant throughout the colony breaks down. The hysteresis in the second order binding has the effect of narrowing down this region. That is why the fully developed switches for the open boundary and the absorbing boundary for the second order binding in Fig. 3 are practically identical.

\section{Discussion}

In order to have a significant effect of the positive-feedback loop the induced production of signal molecules must be much larger than the background production, i.e.

$$
k_{\mathrm{s}} \gg b_{\mathrm{s}}
$$

must be satisfied. Further, in order to be able to fully ignite the feedback

$$
r_{\mathrm{m}} \gtrsim K_{\mathrm{s}}
$$

is required. Provided that growth conditions are such that this is the case, the generic switch will turn on when the size $\Sigma$ becomes sufficiently large to make $r_{\mathrm{a}} \sim \frac{b_{\mathrm{s}}}{k_{\mathrm{s}}} K_{\mathrm{s}}$.

From systematic studies of activities of constitutive promoters in Escherichia coli ${ }^{24,25}$ and chemical composition studies of cells $^{26-28}$ as a function of exponential growth rate we expect that the parameters, e.g. $r_{\mathrm{m}}$, may depend on the growth rate. Further, they may well depend on other physiological parameters like temperature and chemical composition of the environment. 
Thus, in order to establish when a quorum switch can be ignited and sustained, there is a call for studies of transcription rates, RNA turnover, translation rates, and protein turnover under physiological conditions where the culture approaches the stationary phase and starts forming a biofilm.

Typical biofilm thicknesses range from 13-60 $\mu \mathrm{m}$ mean $(34 \pm 10) \mu \mathrm{m}$ in Pseudomonas aeruginosa. ${ }^{19}$ In flow cells (mixed) cultures of Pseudomonas aeruginosa, Pseudomonas fluorescens, Klebsiella pneumoniae, and Stenotrophomonas maltophilia produced biofilms of thickness $(51 \pm 19) \mu \mathrm{m}$ with no flow decreasing to $(37 \pm 12) \mu \mathrm{m}$ in flow. ${ }^{17}$ The horizontal dimension ranged up to $500 \mu \mathrm{m}$. This order of magnitude of the dimension of biofilm structures is confirmed across many bacterial strains, Staphylococcus aureus (30-40 $\mu \mathrm{m}$ thick), ${ }^{20}$ Staphylococcus epidermidis (240-590 $\mu \mathrm{m}$ diameter) ${ }^{18}$ This is to be compared to the degradation range. For acyl homoserine lactones signal molecules at $\mathrm{pH}=7, \mathcal{L}=\sqrt{D / \lambda_{\mathrm{s}}} \sim 7 \mathrm{~mm}$ and at $\mathrm{pH}=8$, $\mathcal{L} \sim 2 \mathrm{~mm}$. Thus the reported ranges of the biofilm dimension give confidence that the thickness is typically below the limit for degradation to be important. The radius $\mathcal{R}$ of a biofilm growing on a surface may, however, become similar to the degradation range $\mathcal{L}$ for signal molecules in large colonies.

Other groups have considered the influence of density and size on ignition of the quorum sensing systems in numerical simulations of specific growth scenarios. ${ }^{29,30}$ While these simulations specifically address the time dependence of the quorum establishment they do not provide analytical insight into conditions required to ignite and sustain a quorum.

The positive feedback is typical for quorum sensors, even in cases where downregulation is desired. However, it may be worth mentioning that explicit negative feedback in the regulator production has been suggested to play a role in the EsaR transcription factor. ${ }^{31,32}$ The analysis presented here, i.e. the dependence on density and geometry, remains valid for this type of regulator. However, where the positive feedback leads to an all-or-none switch, the proposed negative feedback would lead to a smooth downregulation of the maximal transcription factor concentration. This results in an expression like (12) without the feedback switch and with the maximal regulator level suppressed at high concentrations of the regulator.

\section{Conclusion}

We have modeled a generic single-loop quorum sensor with positive feedback. An adequate measure for the size of the quorum sensing ensemble of bacteria has been established and shown to combine the effect of geometric shape, geometric size, cell density, and boundary conditions. Simple expressions for the conditions, (25) and (26), required to sustain an all-or-none quorum switch were established. The results for a thin biofilm suggest that the quorum sensor may act as a flow sensor. When the flow above a biofilm with density $\rho_{\mathrm{v}}$, height $\mathcal{H}$, and radius $\mathcal{R}$, turns off the "size" measure changes from $\mathcal{H}^{2} \rho_{\mathrm{v}}$ to $2 \mathcal{R} \mathcal{H} \rho_{\mathrm{v}}$. For a thin biofilm this can cause the ignition of the quorum merely as a consequence of silencing the flow above the biofilm.
We emphasize that the qualitative behavior of the generic quorum sensor described here is determined by only three dimensionless parameters, $r_{\mathrm{m}} / K_{\mathrm{s}}, k_{\mathrm{s}} / b_{\mathrm{s}}$, and $h$. The influence of the growth state, including growth rate is therefore expected to simply enter through $r_{\mathrm{m}} / K_{\mathrm{s}}$ and $k_{\mathrm{s}} / b_{\mathrm{s}}$. Furthermore, we have shown that $r_{\mathrm{m}} / K_{\mathrm{s}} \gtrsim 1, k_{\mathrm{s}} / b_{\mathrm{s}} \gtrsim 100$, and $h \gtrsim 1$ are sufficient conditions to produce a functioning quorum sensor with an all-or-none switch. The model illuminates the importance of an experimental determination of these parameters under physiological conditions where biofilm colonies are formed.

\section{References}

1 A. Eberhard, J. Bacteriol., 1972, 109, 1101.

2 K. H. Nealson, T. Platt and J. W. Hastings, J. Bacteriol., 1970, 104, 313-322.

3 S. H. Choi and E. P. Greenberg, J. Bacteriol., 1992, 174, 4064-4069.

4 B. L. Hanzelka and E. P. Greenberg, J. Bacteriol., 1995, 177, 815-817.

5 S. Swift, A. V. Karlyshev, E. L. Durant, M. K. Winson, S. R. Chhabra, P. Williams, S. Macintyre and G. S. A. B. Stewart, J. Bacteriol., 1997, 179, 5271-5282.

6 M. J. Lynch, S. Swift, D. F. Kirke, C. W. Keevil, C. E. R. Dodd and P. Williams, Environ. Microbiol., 2002, 4, 18-28.

7 D. F. Kirke, S. Swift, M. J. Lynch and P. Williams, FEMS Microbiol. Lett., 2004, 241, 109-117.

8 M. J. Bottomley, E. Muraglia, R. Bazzo and A. Carfi, J. Biol. Chem., 2007, 282, 13592-13600.

9 C. Fuqua, S. C. Winans and E. P. Greenberg, Annu. Rev. Microbiol., 1996, 50, 727-753.

10 M. I. Moré, L. D. Finger, J. L. Stryker, C. Fuqua, A. Eberhard and S. C. Winans, Science, 1996, 272, 1655-1658.

11 A. V. Hill, Proc. Physiol. Soc., 1910, iv-vii.

12 M. Welch, J. Gross, J. T. Hodgkinson, D. R. Spring and T. Sams, Biochem., 2013, 52, 4433-4438.

13 M. Laurent and N. Kellershohn, Trends Biochem. Sci., 1999, 24, 418-422.

14 J. B. Andersen, C. Sternberg, L. K. Poulsen, S. P. Bjorn, M. Givskov and S. Molin, Appl. Environ. Microbiol., 1998, 64, 2240-2246.

15 M. Welch, D. E. Todd, N. A. Whitehead, S. J. McGowan, B. W. Bycroft and G. P. Salmond, EMBO J., 2000, 19, 631-641.

16 C. Garde, T. Bjarnsholt, M. Givskov, T. H. Jakobsen, M. Hentzer, A. Claussen, K. Sneppen, J. Ferkinghoff-Borg and T. Sams, J. Mol. Biol., 2010, 396, 849-857.

17 P. Stoodley, Z. Lewandowski, J. D. Boyle and H. M. LappinScott, Biotechnol. Bioeng., 1999, 65, 83-92.

18 C. A. Fux, J. W. Costerton, P. S. Stewart and P. Stoodley, Trends Microbiol., 2005, 13, 34-40.

19 P. S. Stewart, B. M. Peyton, W. J. Drury and R. Murga, Appl. Environ. Microbiol., 1993, 59, 327-329.

20 K. K. Jefferson, D. A. Goldmann and G. B. Pier, Antimicrob. Agents Chemother., 2005, 49, 2467-2473. 
21 A. L. Schaefer, B. L. Hanzelka, M. R. Parsek and E. P. Greenberg, Methods Enzymol., 2000, 305, 288-301.

22 A. R. Horswill, P. Stoodley, P. S. Stewart and M. R. Parsek, Anal. Bioanal. Chem., 2007, 387, 371-380.

23 E. A. Yates, B. Philipp, C. Buckley, S. Atkinson, S. R. Chhabra, R. E. Sockett, M. Goldner, Y. Dessaux, M. Cámara, H. Smith and P. Williams, Infect. Immun., 2002, 70, 5635-5646.

24 S.-T. Liang, M. Bipatnath, Y.-C. Xu, S.-L. Chen, P. Dennis, M. Ehrenberg and H. Bremer, J. Mol. Biol., 1999, 292, 19-37.

25 S.-T. Liang, Y.-C. Xu, P. Dennis and H. Bremer, J. Bacteriol., 2000, 182, 3037-3044.

26 H. Bremer and P. P. Dennis, in Escherichia coli and Salmonella: Cellular and Molecular Biology, ed. F. C. Neidhart, ASM Press, Washington, DC, 1996, ch. 97: Modulation of chemical composition and other parameters of the cell by growth rate.
27 S. Klumpp, Z. Zhang and T. Hwa, Cell, 2009, 139, 1366-1375. 28 M. Scott, C. W. Gunderson, E. M. Mateescu, Z. Zhang and T. Hwa, Science, 2010, 330, 1099-1102.

29 P. Nilsson, A. Olofsson, M. Fagerlind, T. Fagerstrom, S. Rice, S. Kjelleberg and P. Steinberg, J. Mol. Biol., 2001, 309, 631-640.

30 M. E. Parent, C. E. Snyder, N. D. Kopp and D. Velegol, Colloids Surf., B: Biointerfaces, 2008, 62, 180-187.

31 C.-S. Tsai and S. C. Winans, Mol. Microbiol., 2010, 77, 1072-1082.

32 S. B. von Bodman, D. R. Majerczak and D. L. Coplin, Proc. Natl. Acad. Sci. U. S. A., 1998, 95, 7687-7692.

33 N. H. Asmar, Partial differential equations with Fourier series and boundary value problems, Pearson Prentice Hall, Upper Saddle River, NJ, 2005, pp. 1-690, pp. A1-A112. 